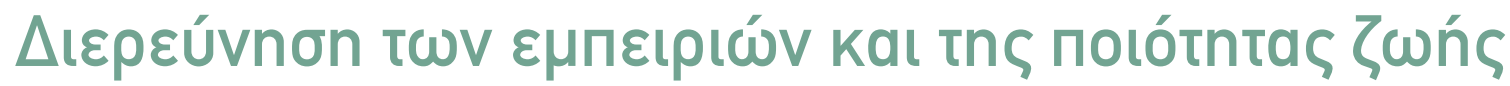

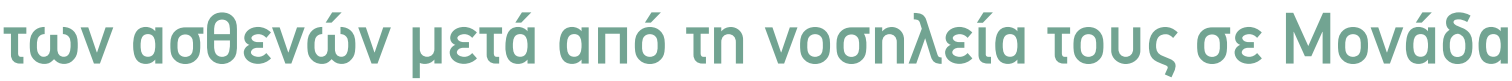

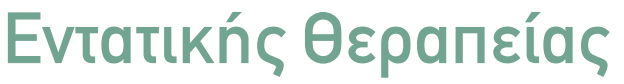

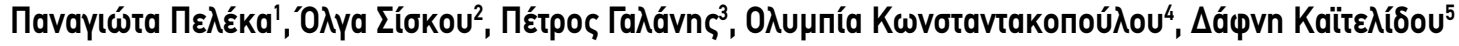

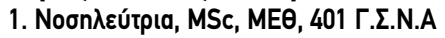

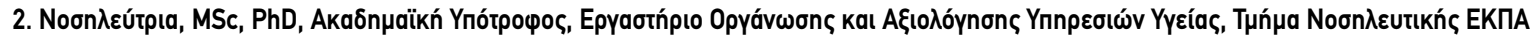

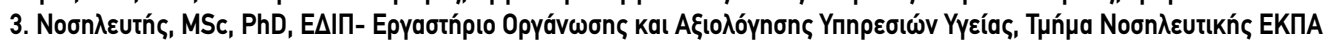

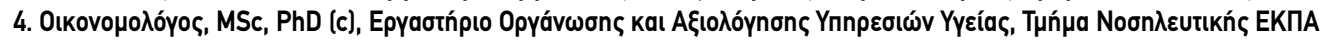

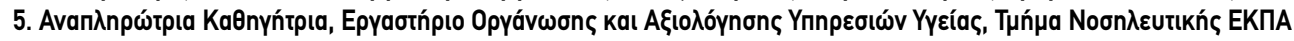

\section{ПEPIAHWH}

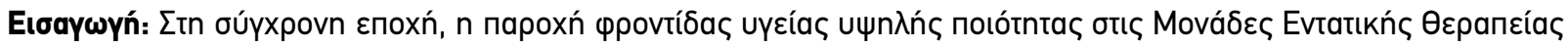

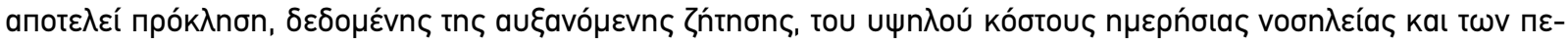

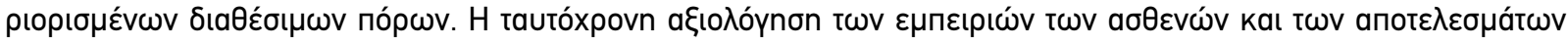

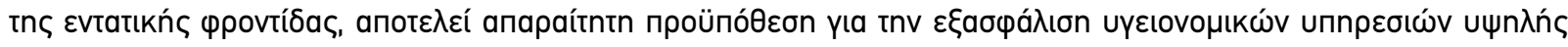
пolótntac.

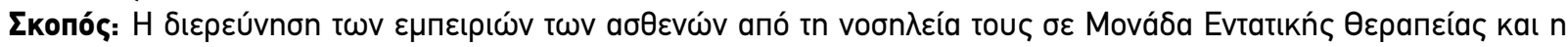

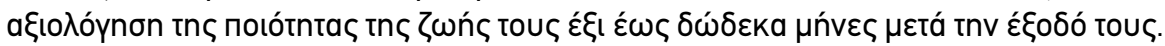

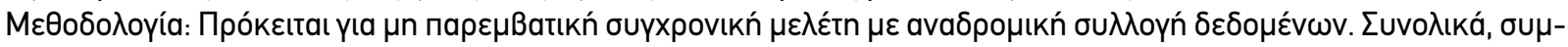

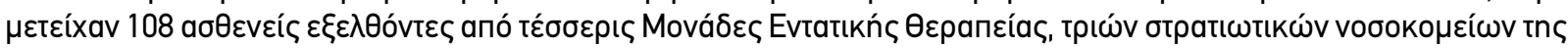

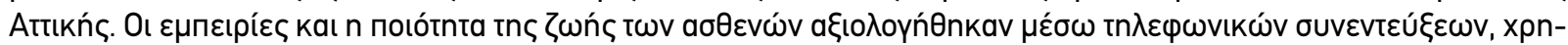

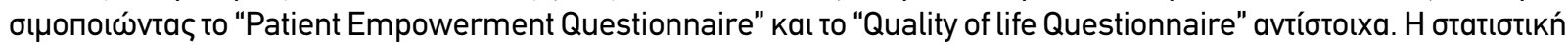

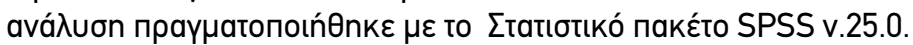

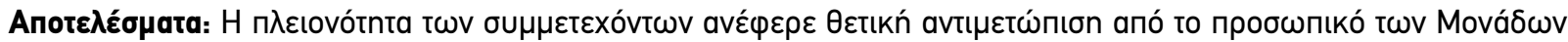

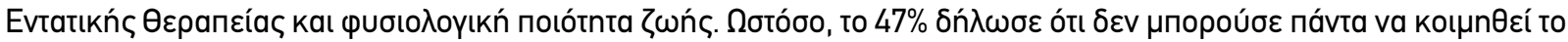

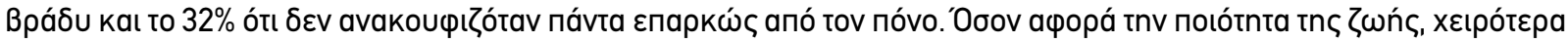

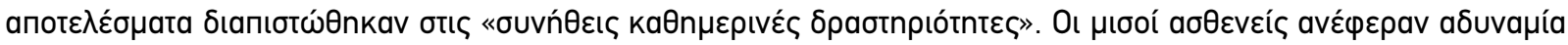

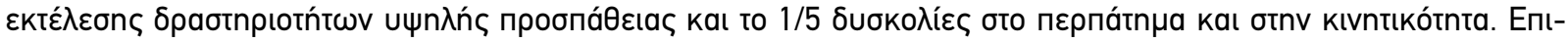

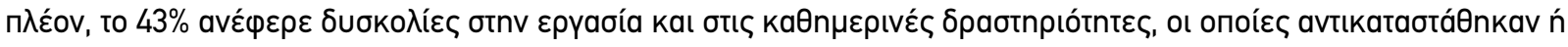

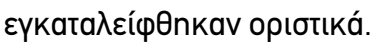

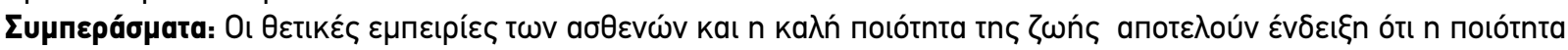

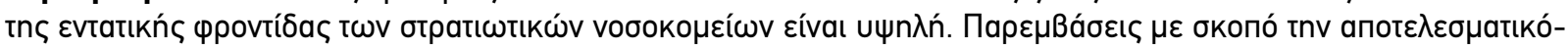

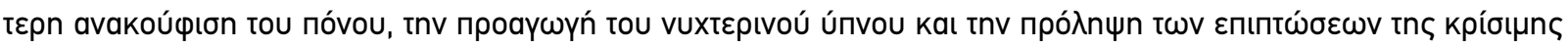

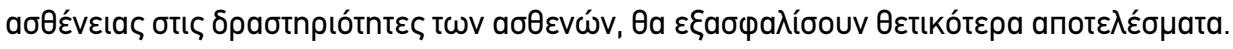

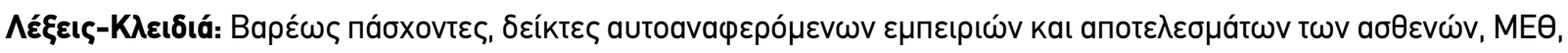

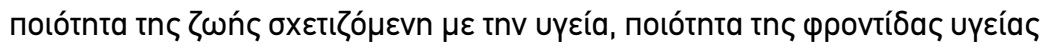

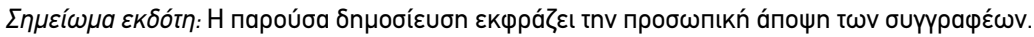

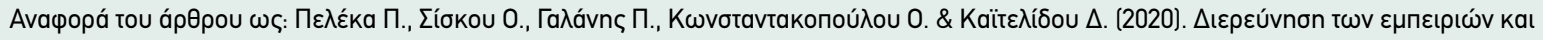

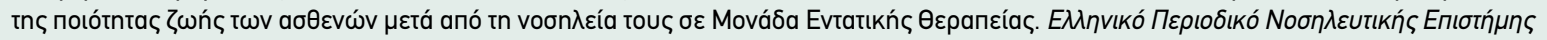
13(4): 51-59, https://doi.org/10.24283/hjns.202045 


\section{EPEYNHTIKA APOPA}

\section{BAEIKA EHMEIA}

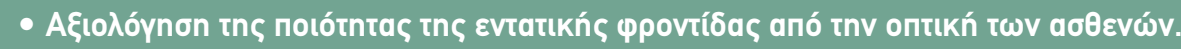

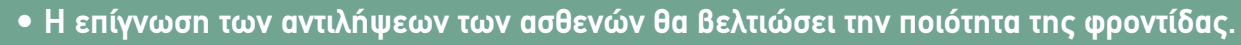

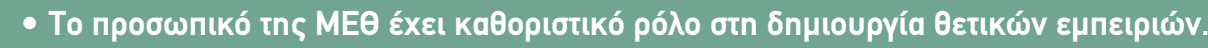

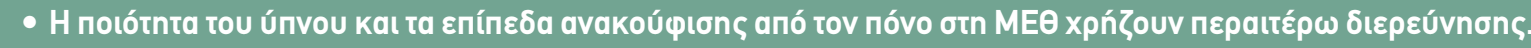

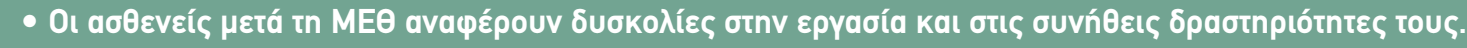

\section{H}

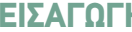

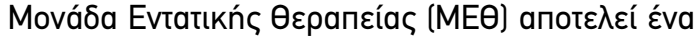

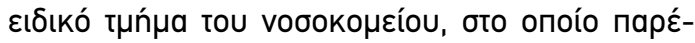

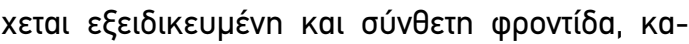

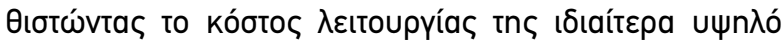

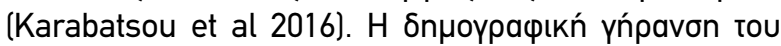

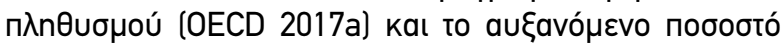

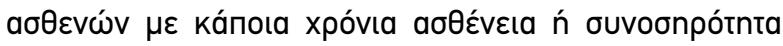

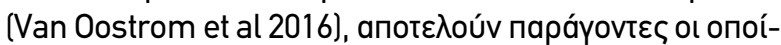

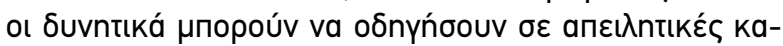

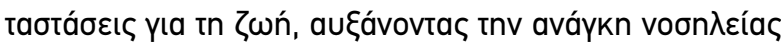

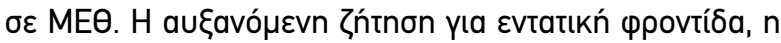

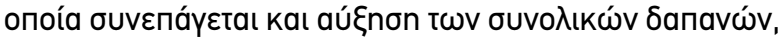

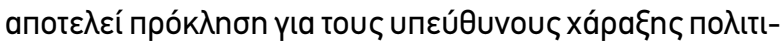

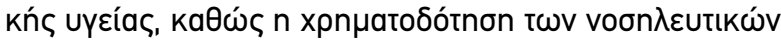

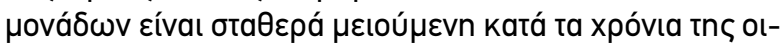

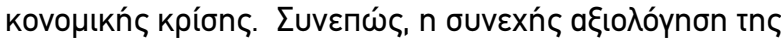

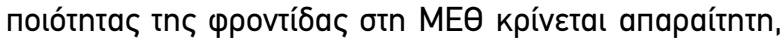

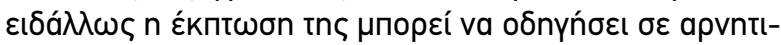

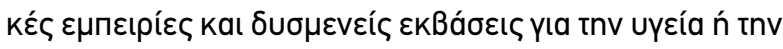

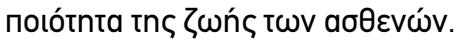

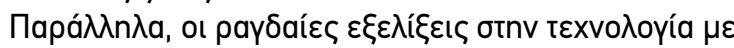

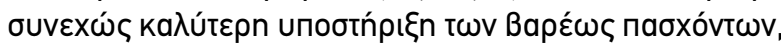

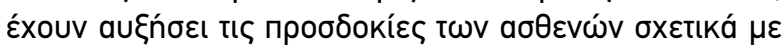

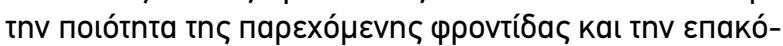

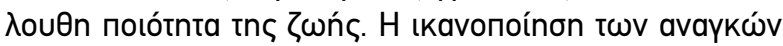

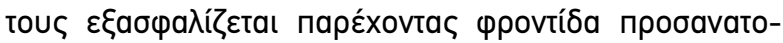

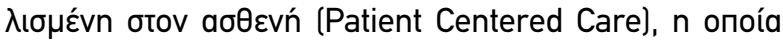

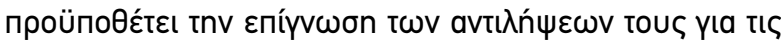

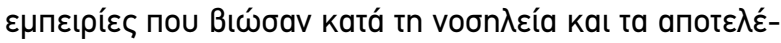

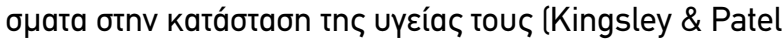
2017).

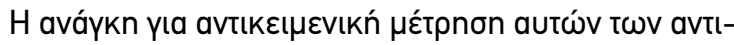

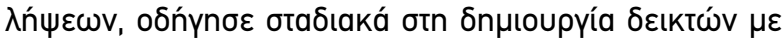

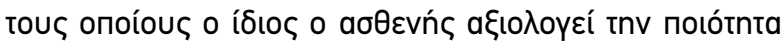

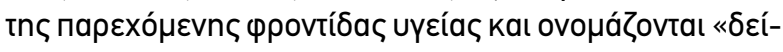

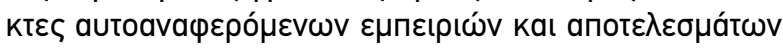

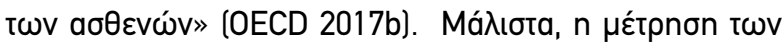

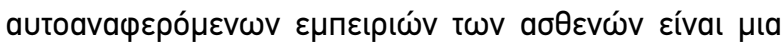

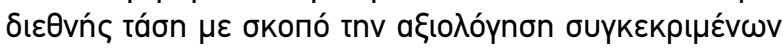

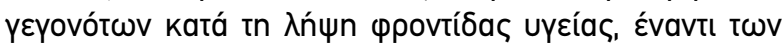

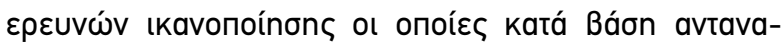

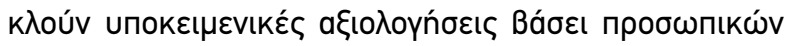

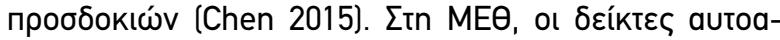

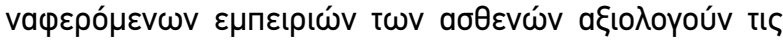

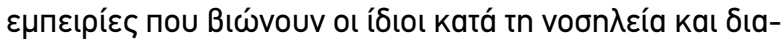

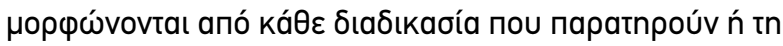

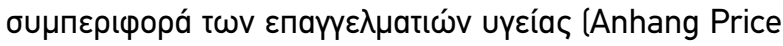

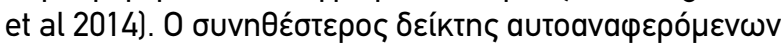

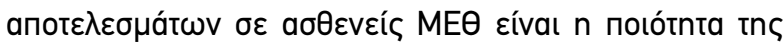

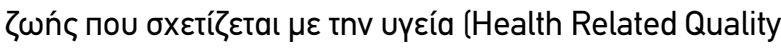
of Life) (McKinley et al 2016, Ferrand et al 2019).

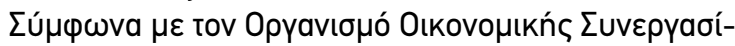

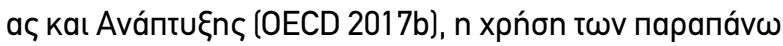

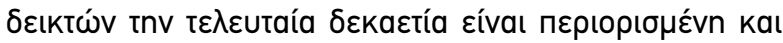

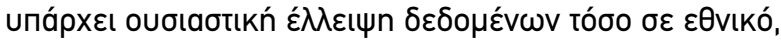

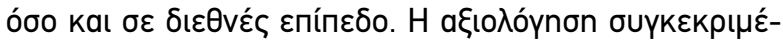

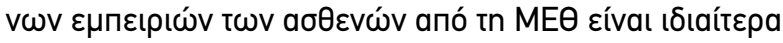

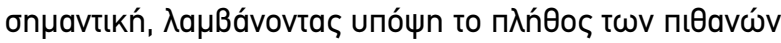

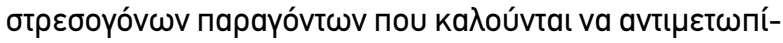

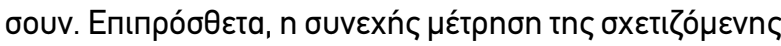

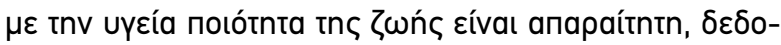

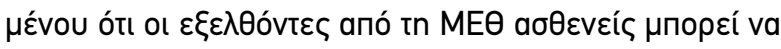

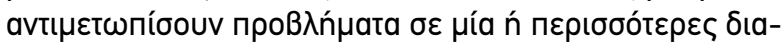

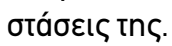

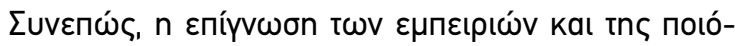

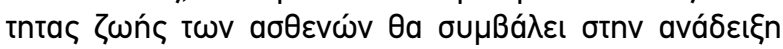

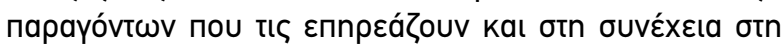

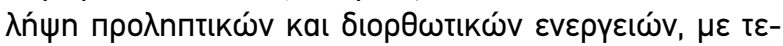

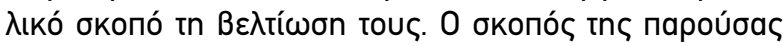
$\mu \varepsilon \lambda \varepsilon ́$ tn

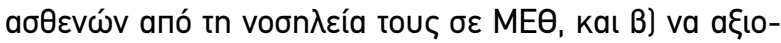

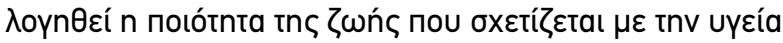

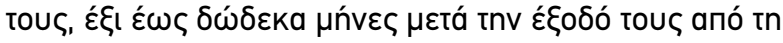
MEQ.

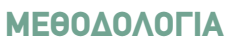

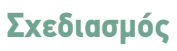

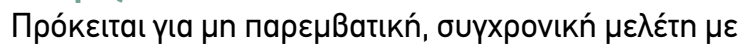

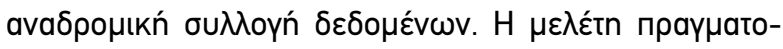

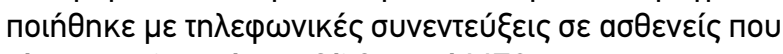

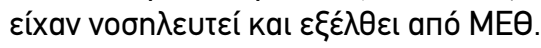

\section{$\triangle$ Eípu a}

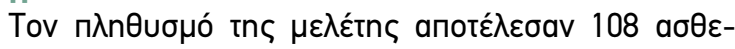

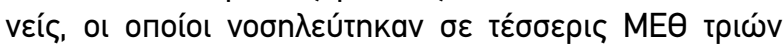




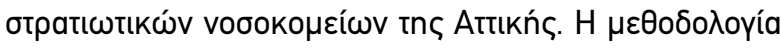

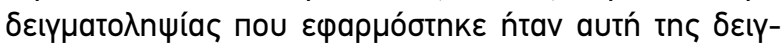

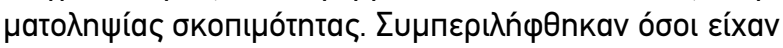

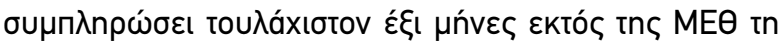

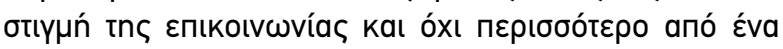

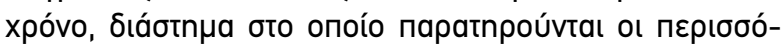

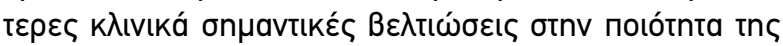

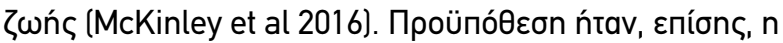

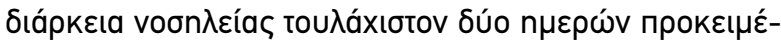

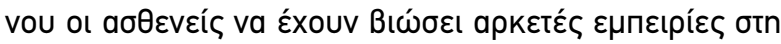

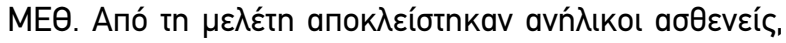

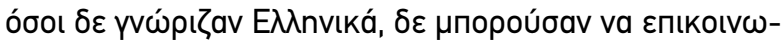

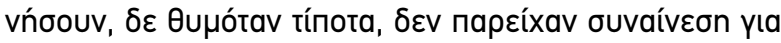

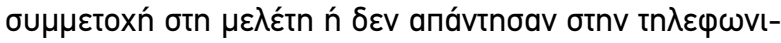

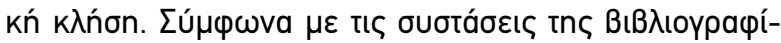

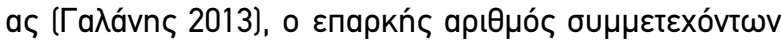

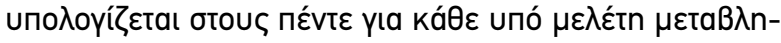

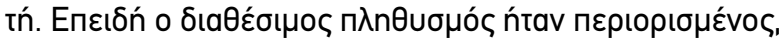

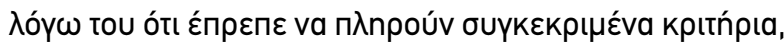

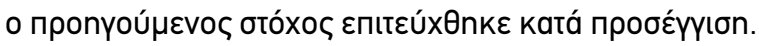

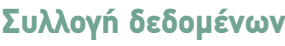

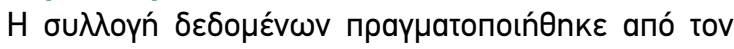

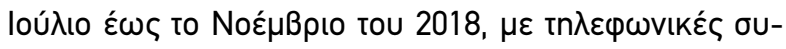

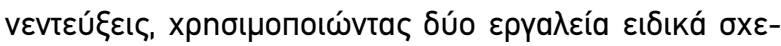

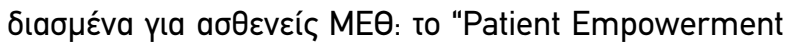
Questionnaire" (Wahlin et al 2017) kaı to "Quality of Life Questionnaire" (Fernandez et al 1996) kaı үıа та опоía

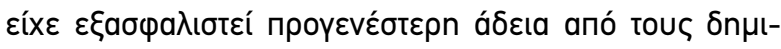
oupyoúc.

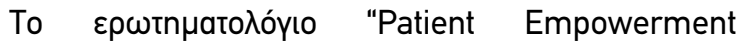

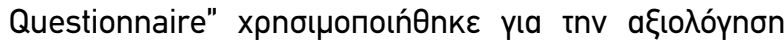

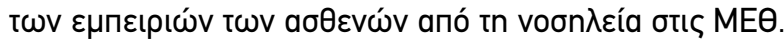

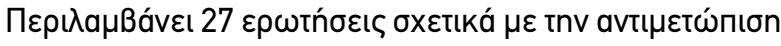

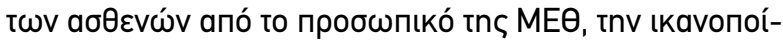

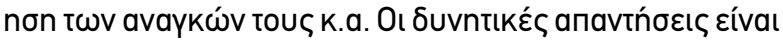

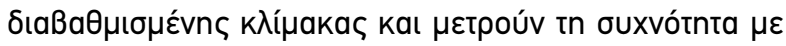

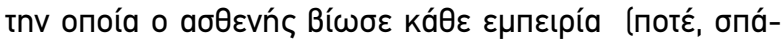

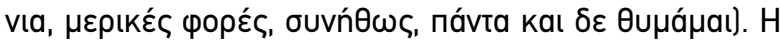

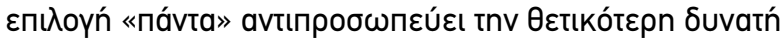

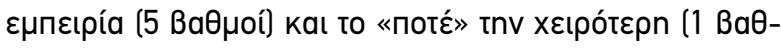
Hóc).

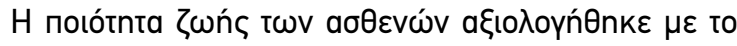

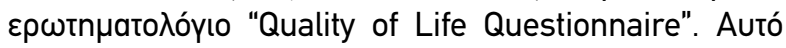

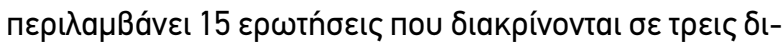

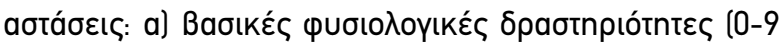

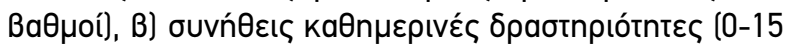

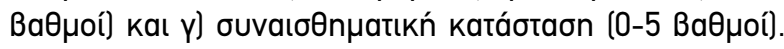

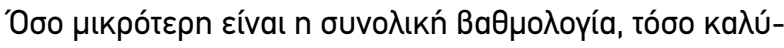

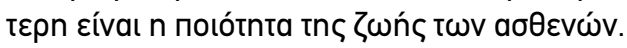

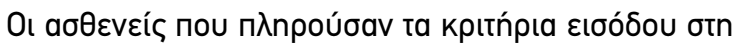

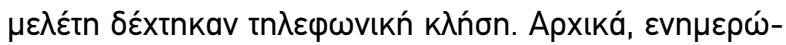

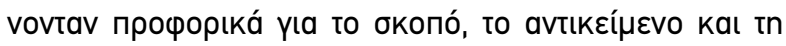

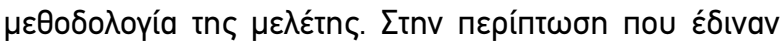

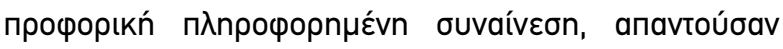

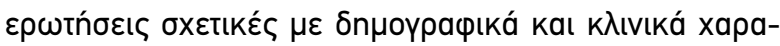

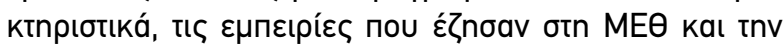

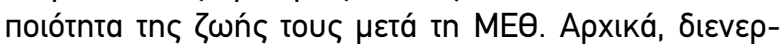

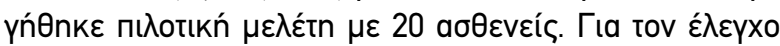

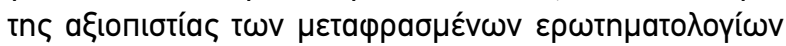

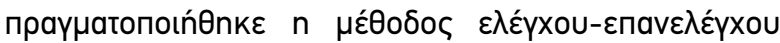

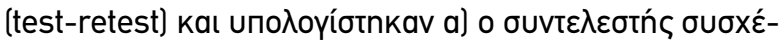

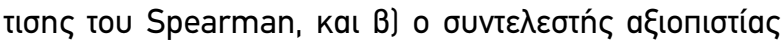
Cronbach alpha.

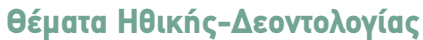

Oı $\Delta \rho$. Wahlin, $\Delta \rho$. Vazquez-Mata kaı $\Delta \rho$. Fernandez

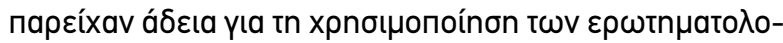

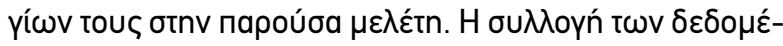

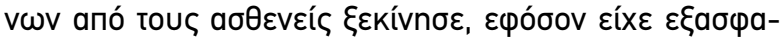

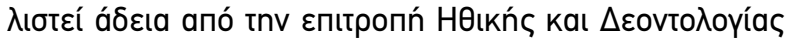

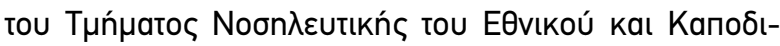

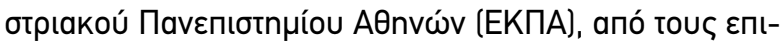

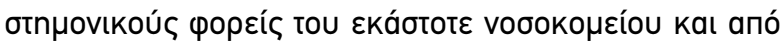

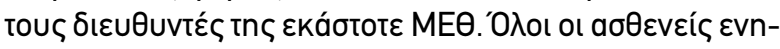

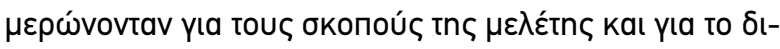

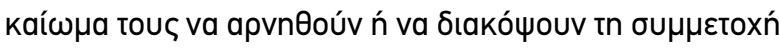

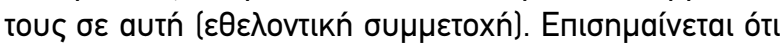

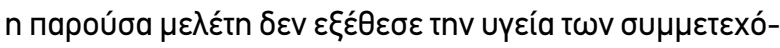

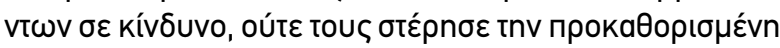

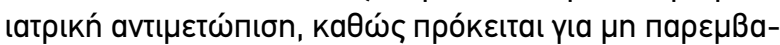

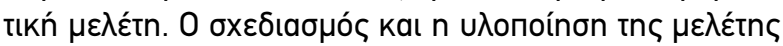

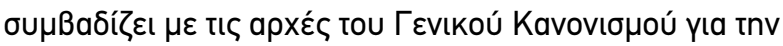

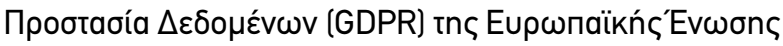

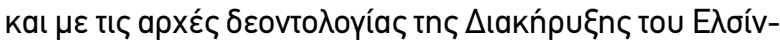
Kı (World Medical Association Declaration of Helsinki). H

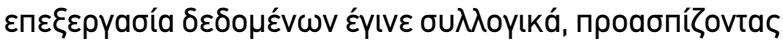

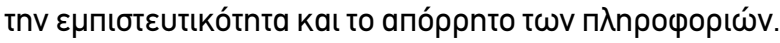

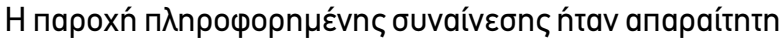

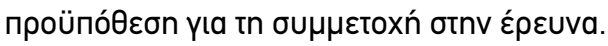

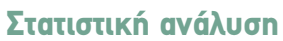

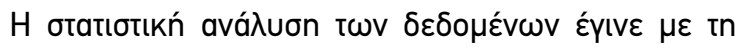

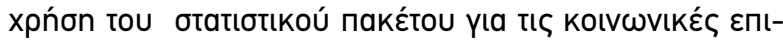

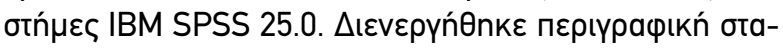

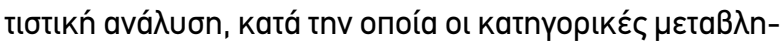

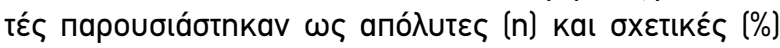

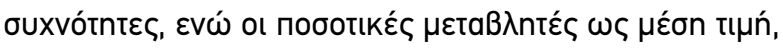

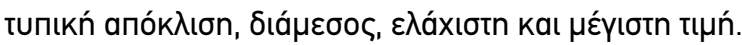

\section{AПOTEАEЕMATA}

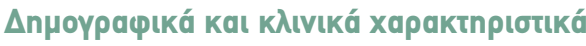

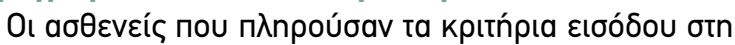

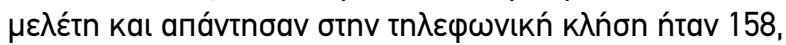

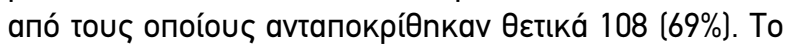

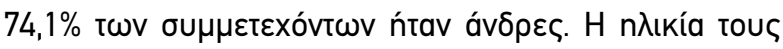




\section{EPEYNHTIKA APOPA}

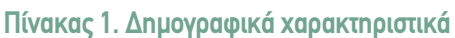

\begin{tabular}{|c|c|c|}
\hline Фúlo & $\mathbf{N}$ & $\%$ \\
\hline 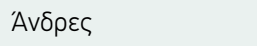 & 80 & 74,1 \\
\hline Гuvaíkعৎ & 28 & 25,9 \\
\hline \multicolumn{3}{|l|}{ H入ıкía } \\
\hline 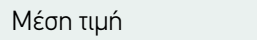 & \multicolumn{2}{|c|}{52,42} \\
\hline 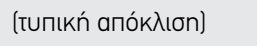 & \multicolumn{2}{|c|}{$(20,302)$} \\
\hline 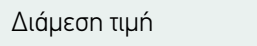 & \multicolumn{2}{|c|}{55} \\
\hline 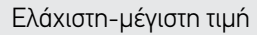 & \multicolumn{2}{|c|}{$19-89$} \\
\hline
\end{tabular}

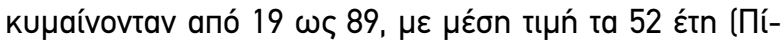

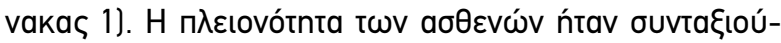

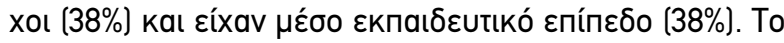

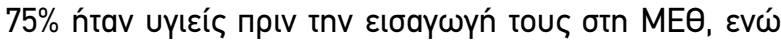

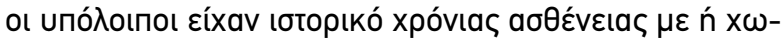

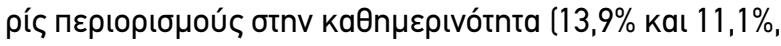

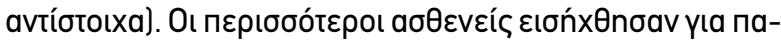

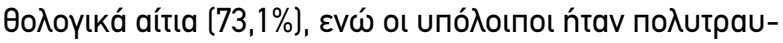

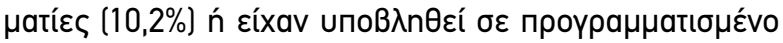

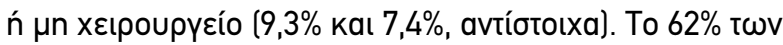

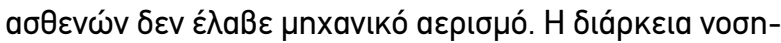

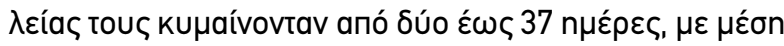

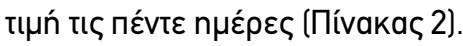

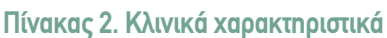

\begin{tabular}{|c|c|c|}
\hline Характnрібткќ́ & $\mathbf{N}$ & $\%$ \\
\hline \multicolumn{3}{|l|}{ 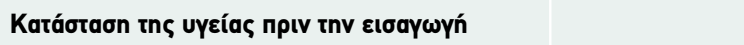 } \\
\hline Yyı́ns & 81 & 75 \\
\hline 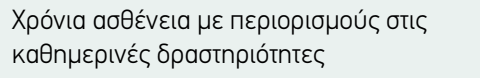 & 15 & 13,9 \\
\hline 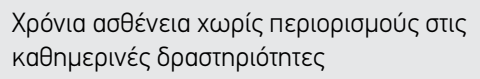 & 12 & 11,1 \\
\hline \multicolumn{3}{|l|}{ 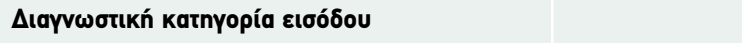 } \\
\hline ПаӨо入оүıкá aítıa & 79 & 73,1 \\
\hline 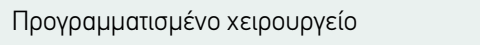 & 10 & 9,3 \\
\hline 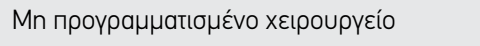 & 8 & 7,4 \\
\hline 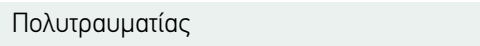 & 11 & 10,2 \\
\hline \multicolumn{3}{|l|}{ 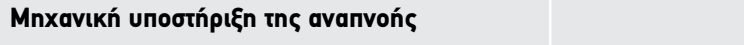 } \\
\hline 'Oxı & 67 & 62 \\
\hline Nal & 41 & 38 \\
\hline \multicolumn{3}{|l|}{ 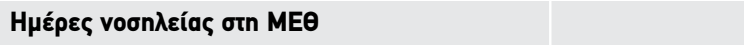 } \\
\hline 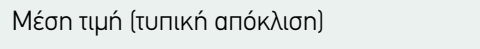 & \multicolumn{2}{|c|}{$5,20(5,714)$} \\
\hline 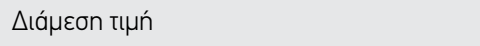 & \multicolumn{2}{|c|}{3,00} \\
\hline 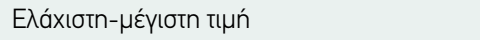 & \multicolumn{2}{|c|}{$2-37$} \\
\hline
\end{tabular}

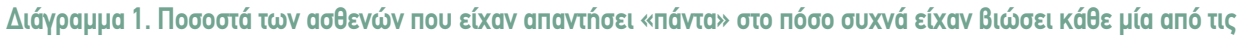

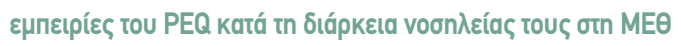

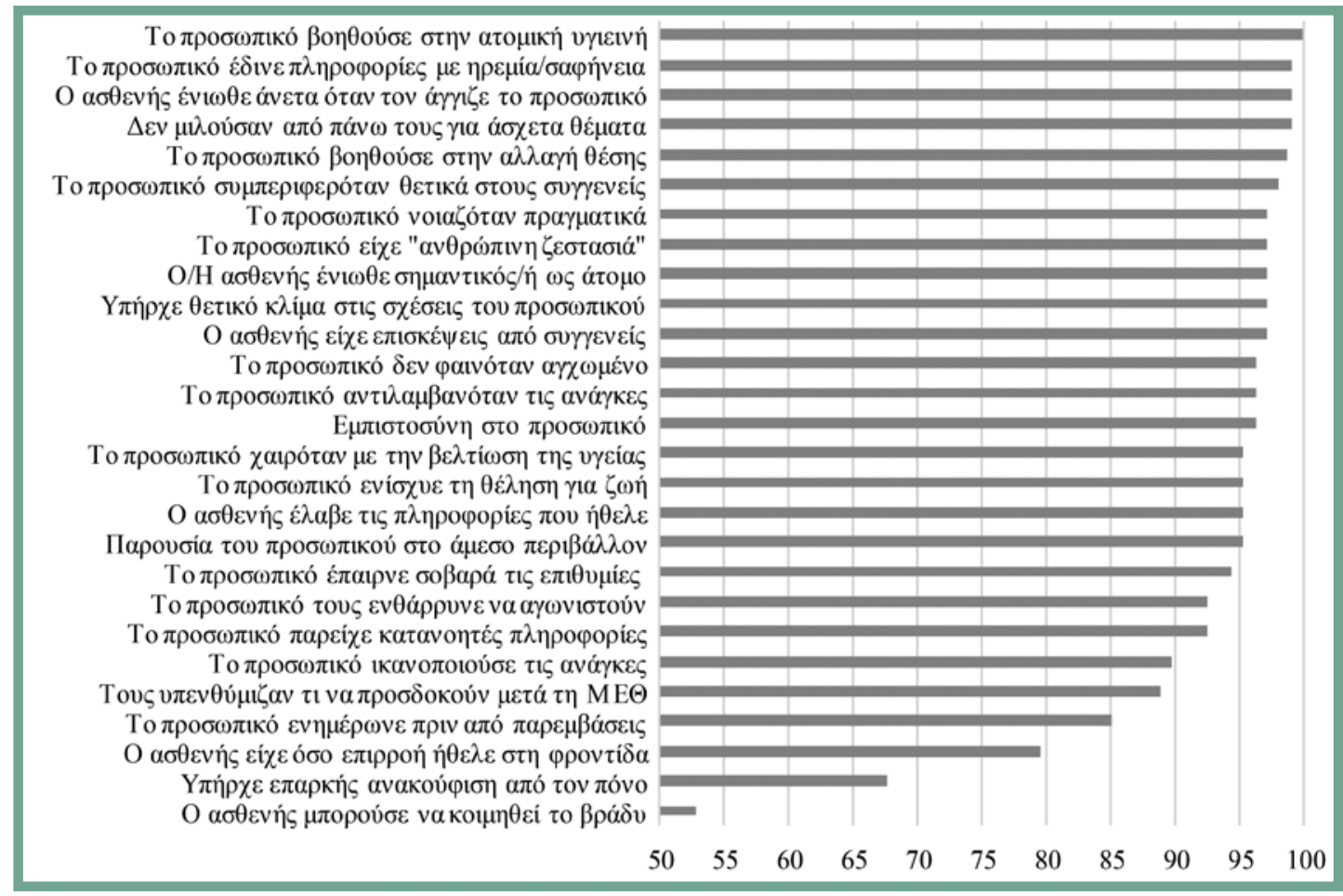




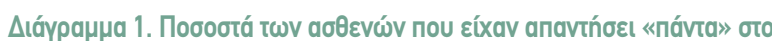

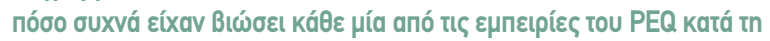

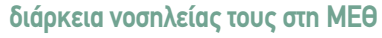

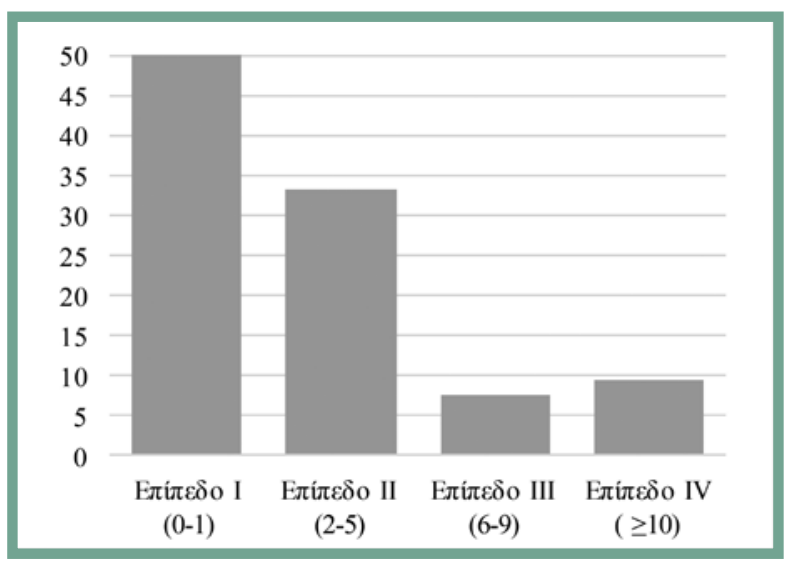

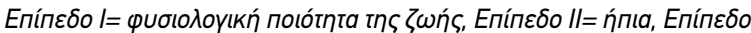

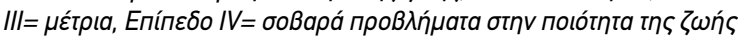

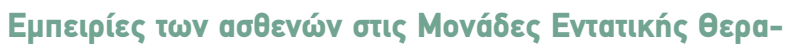
пвías

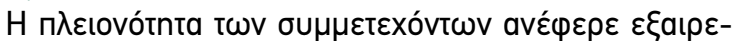

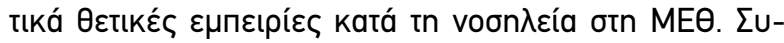

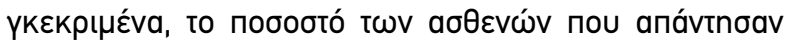

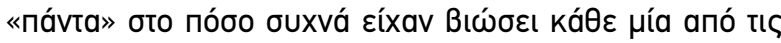

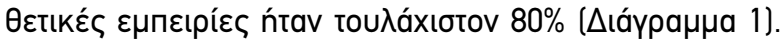

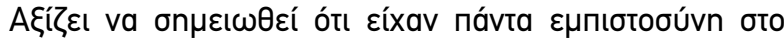

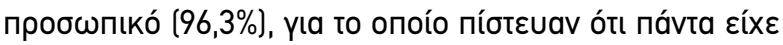

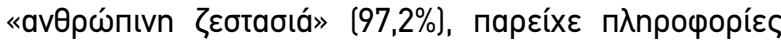

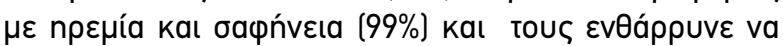

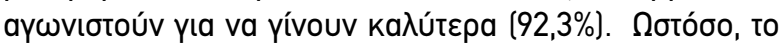

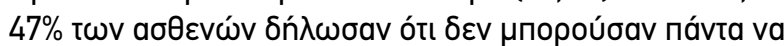

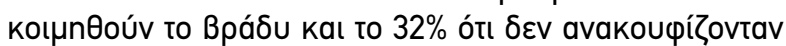

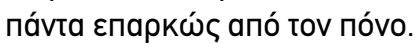

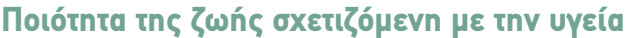

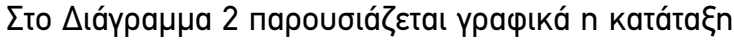

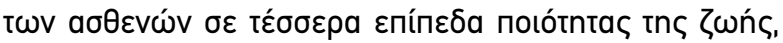

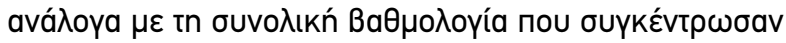

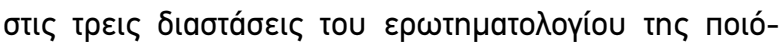

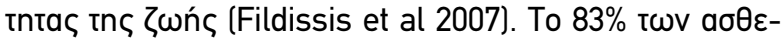

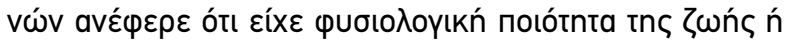

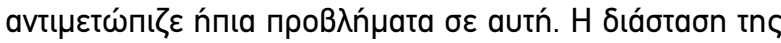

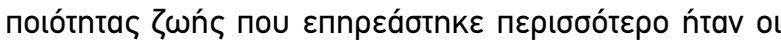

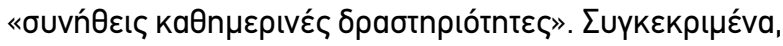

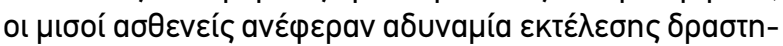

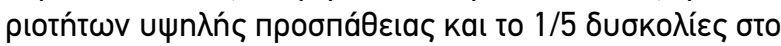

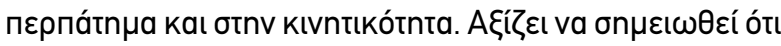

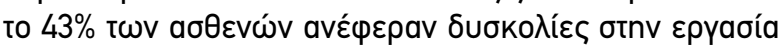

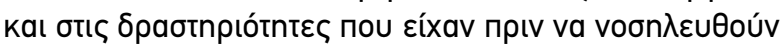

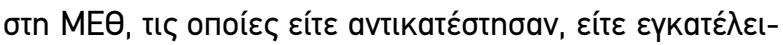

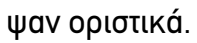

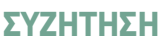

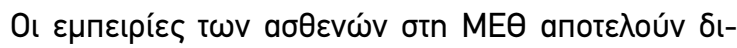

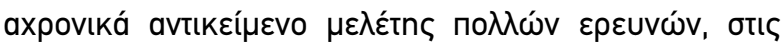

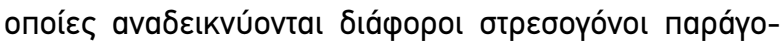

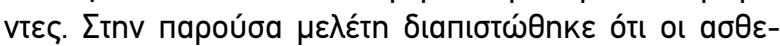

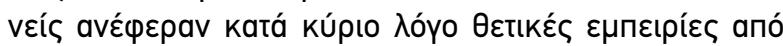

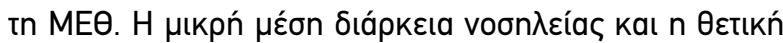

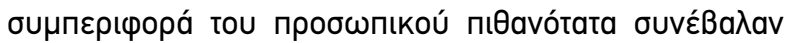

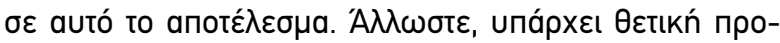

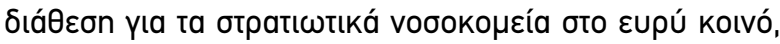
kaӨ

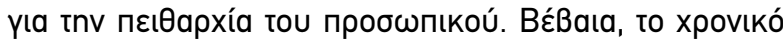

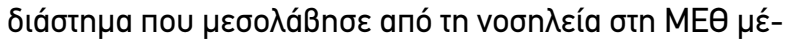

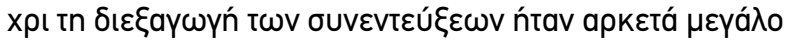

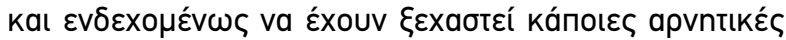

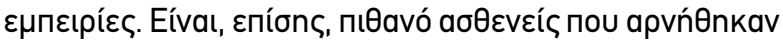

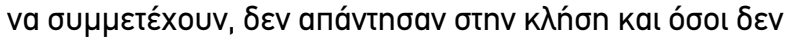

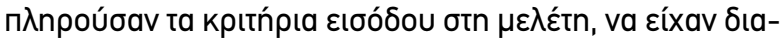

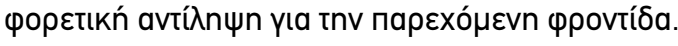

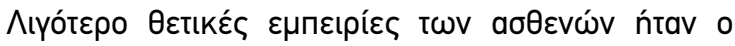

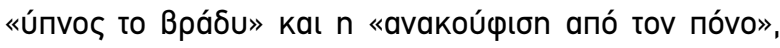

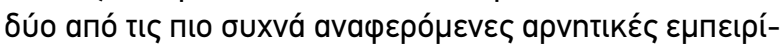

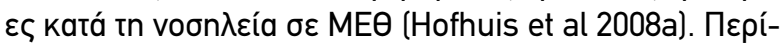

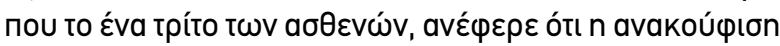

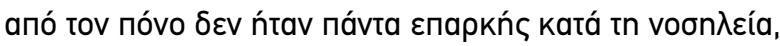

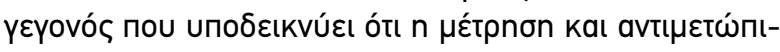

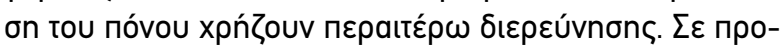

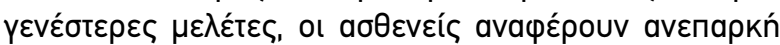

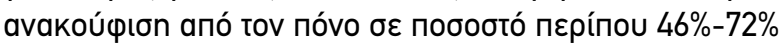
(Hofhuis et al 2008a, Usta et al 2016). $\Sigma \varepsilon$ avtí日 $\varepsilon \sigma n, \sigma$ otn $\mu \varepsilon-$

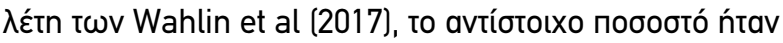

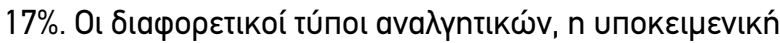

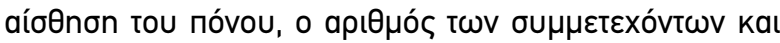

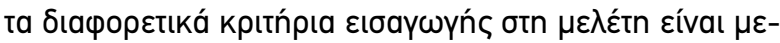

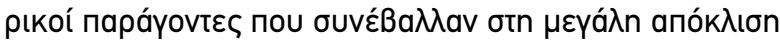

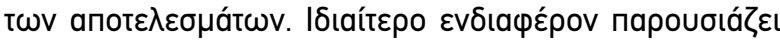

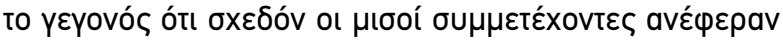

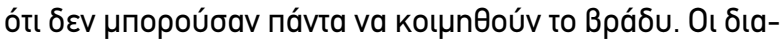

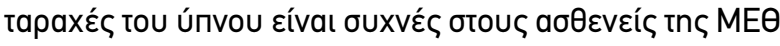

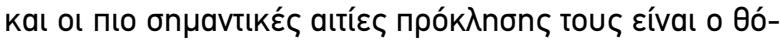
pußoৎ kaı o nóvoৎ (Wahlin et al 2017, Stewart et al 2017).

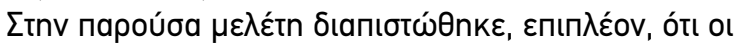

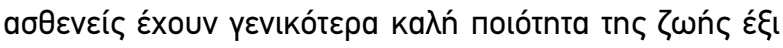

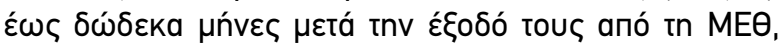

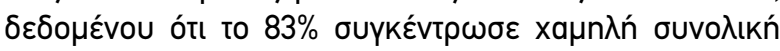

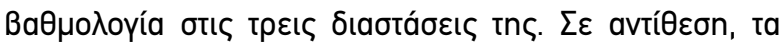

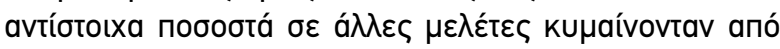

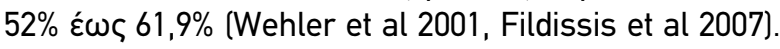

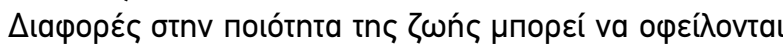

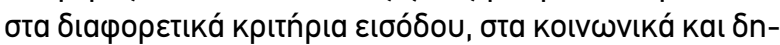

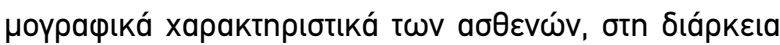

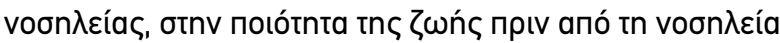

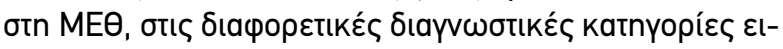




\section{EPEYNHTIKA APӨPA}

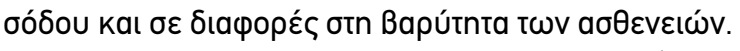

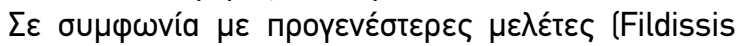

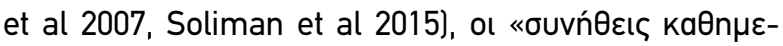

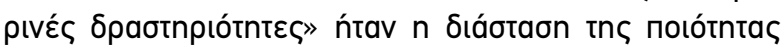

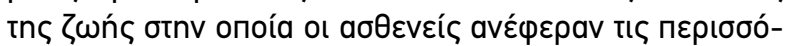

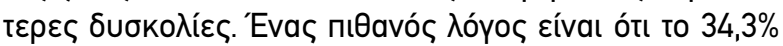

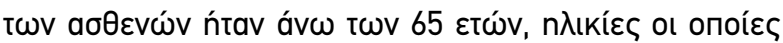

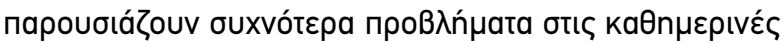

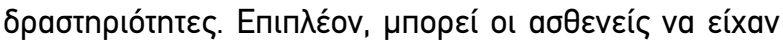

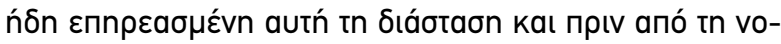

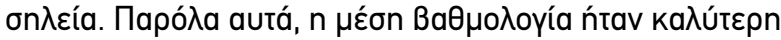

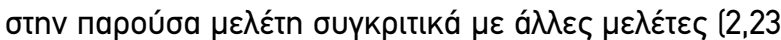

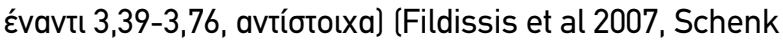
et al 2012).

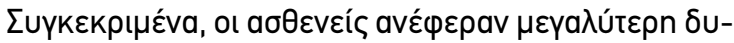

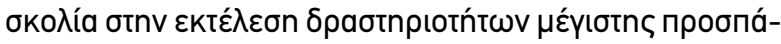

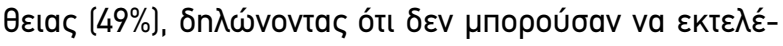

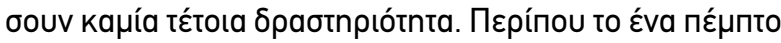

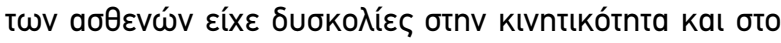

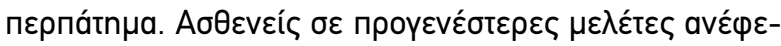

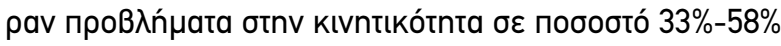
(Pandian et al 2013, Busico et al 2016).

A

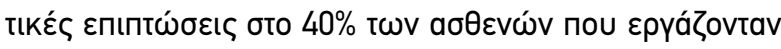

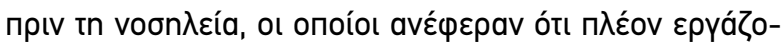

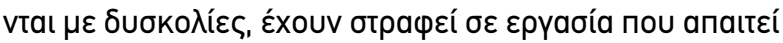

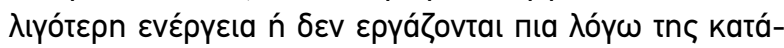

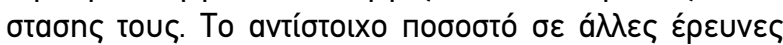

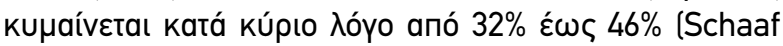

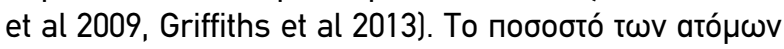

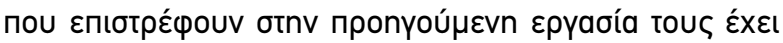

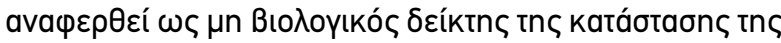

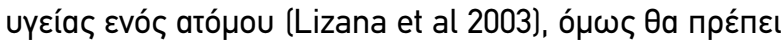

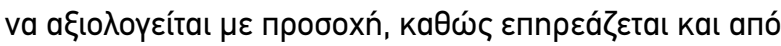

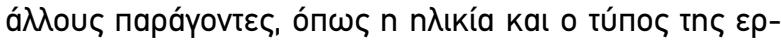
yaoías.

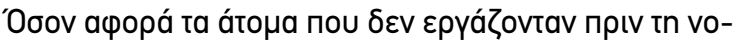

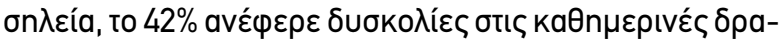

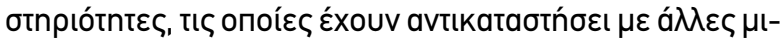

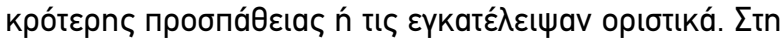

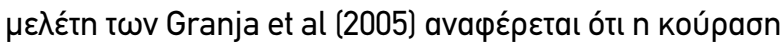

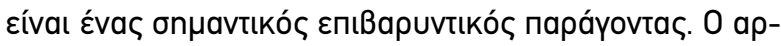

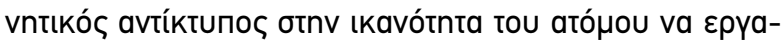

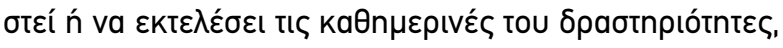

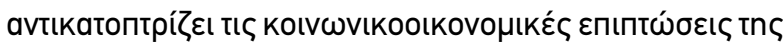

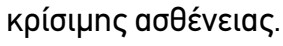

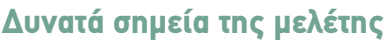

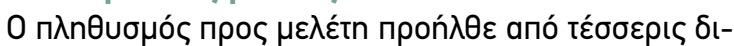

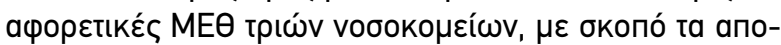

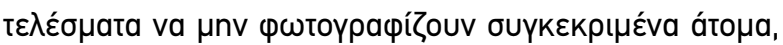

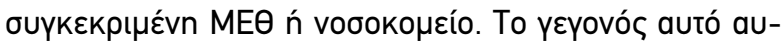

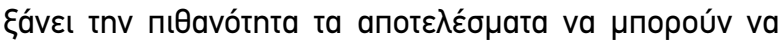

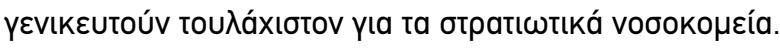

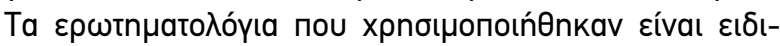

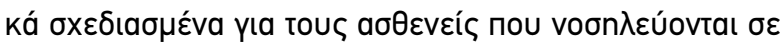

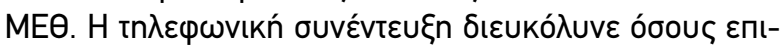

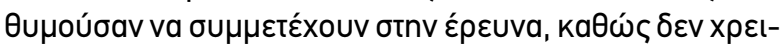

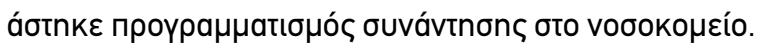

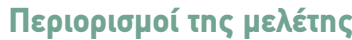

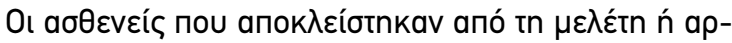

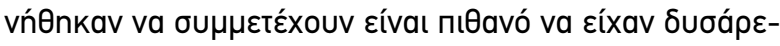

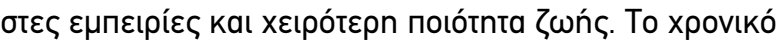

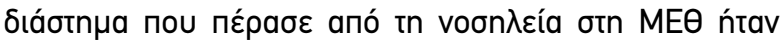

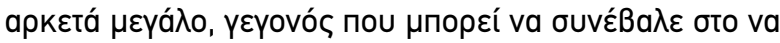

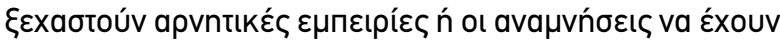

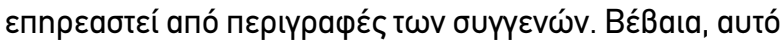

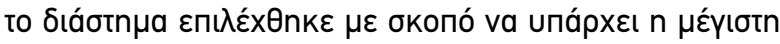

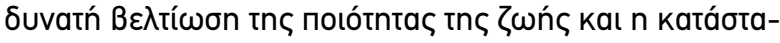

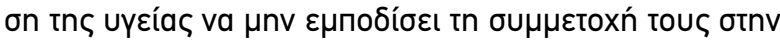

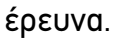

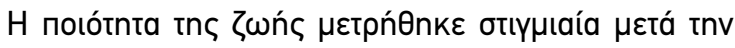

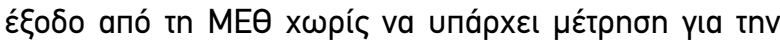

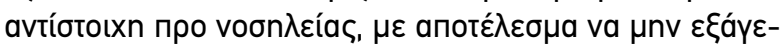

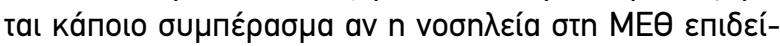

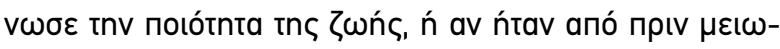

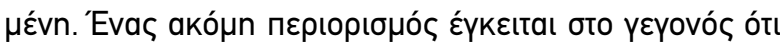

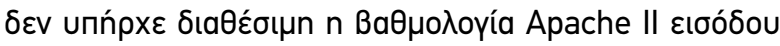

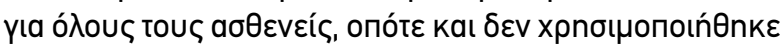

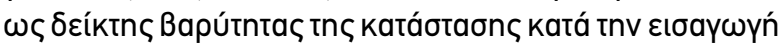
TOUৎ.

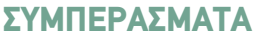

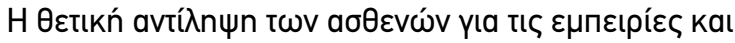

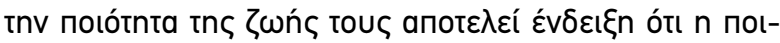

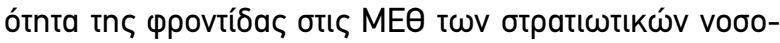

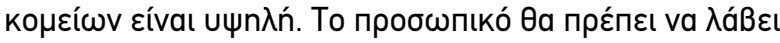

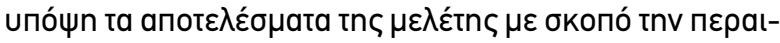

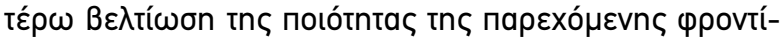

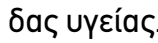

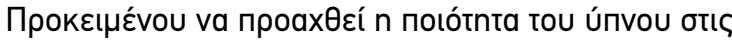

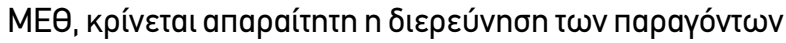

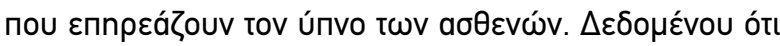

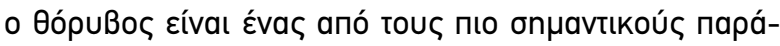

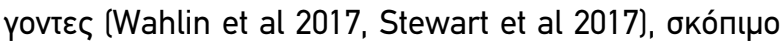

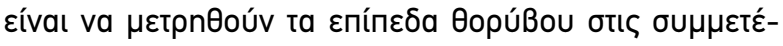

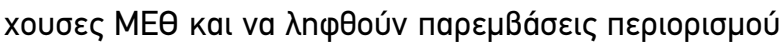

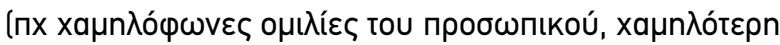

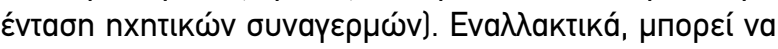

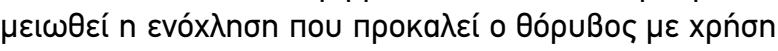

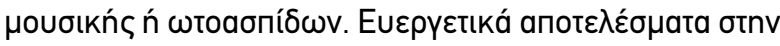

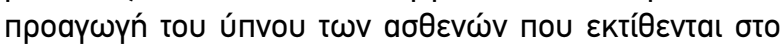

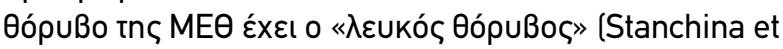
al 2005).

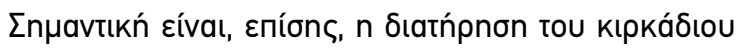




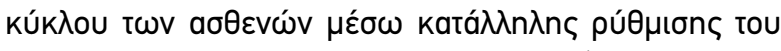

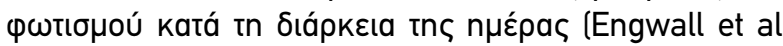

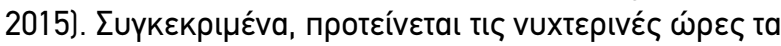

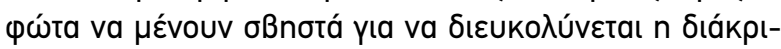

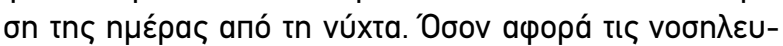

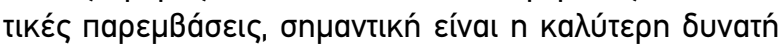

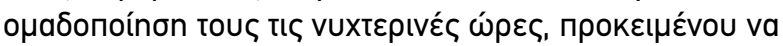

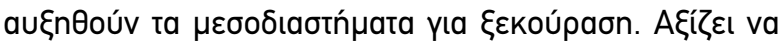

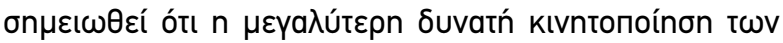

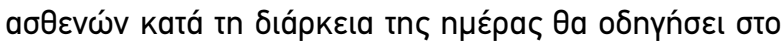

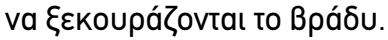

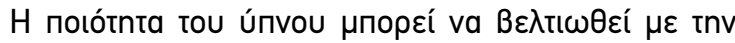

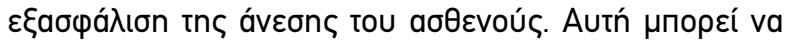

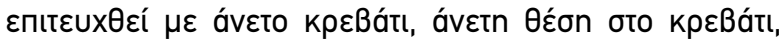

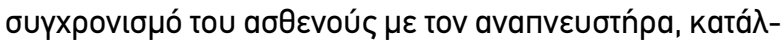

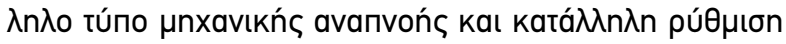

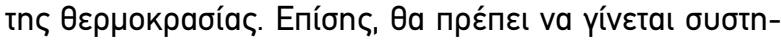

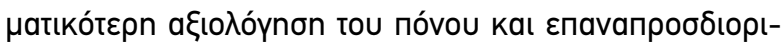

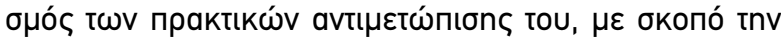

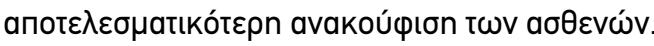

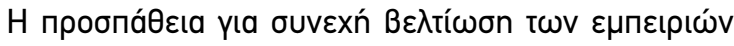

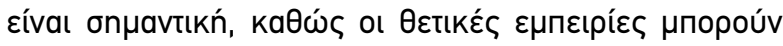

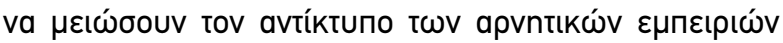

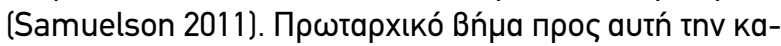

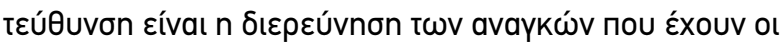

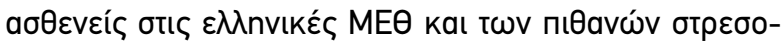

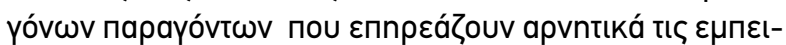

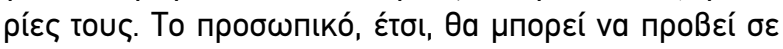

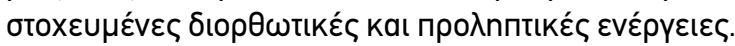

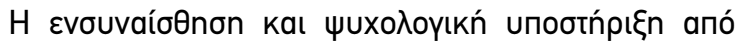

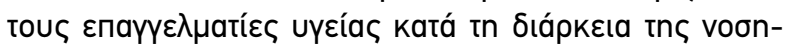

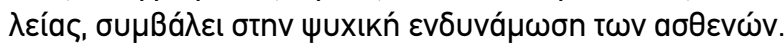

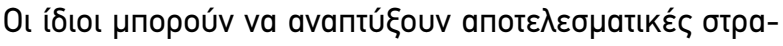

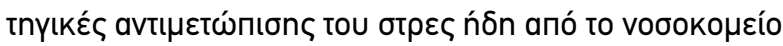

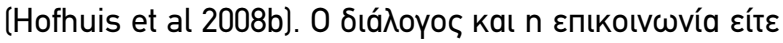

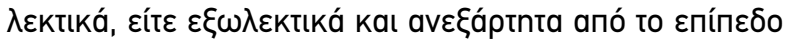

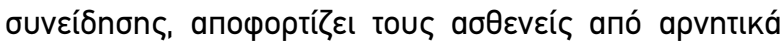

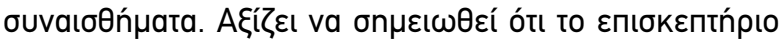

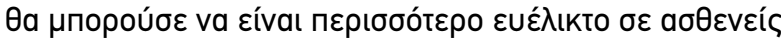

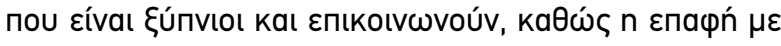

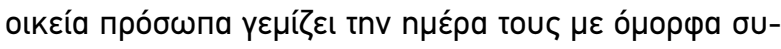

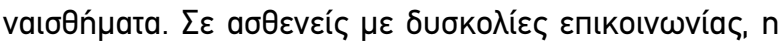

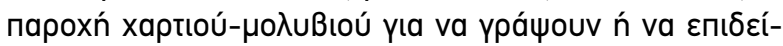

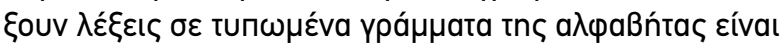

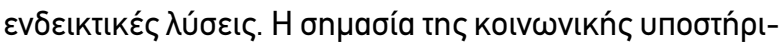

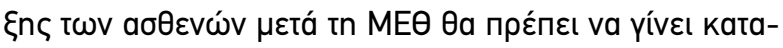

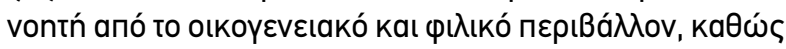

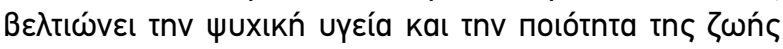

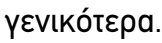

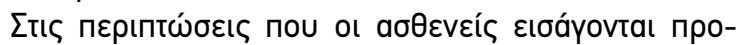

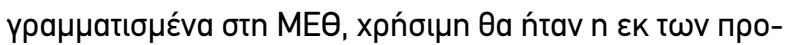

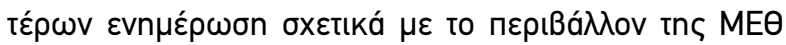

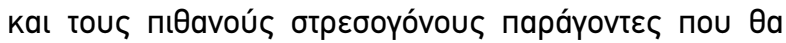

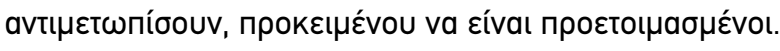

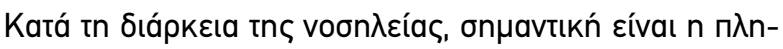

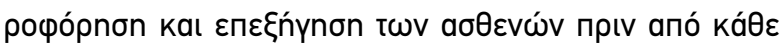

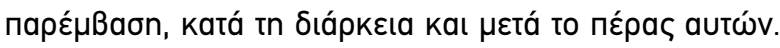

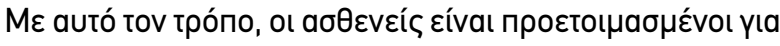

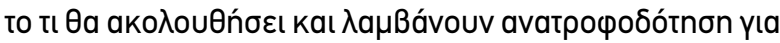

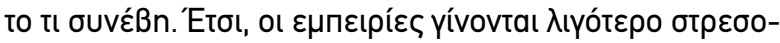

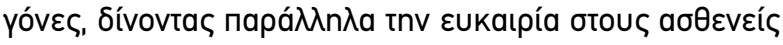

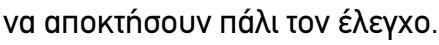

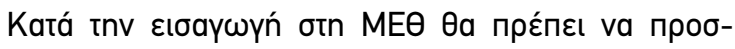

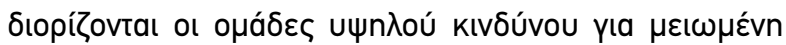

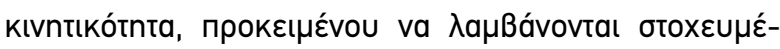

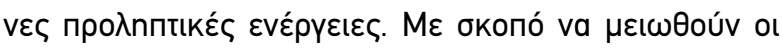

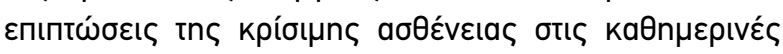

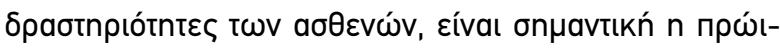

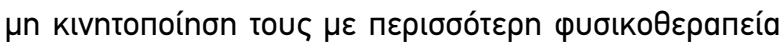

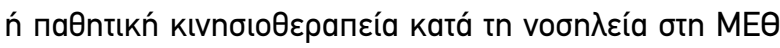

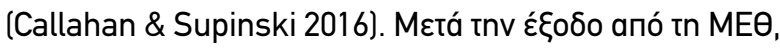

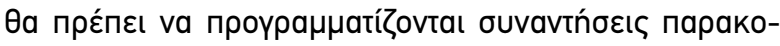

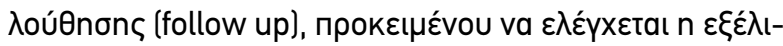

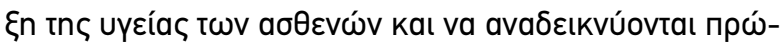

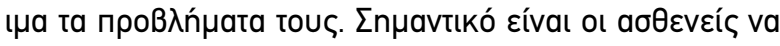

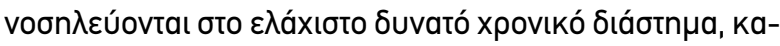

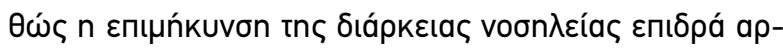

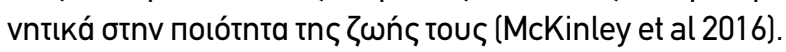

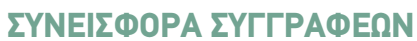

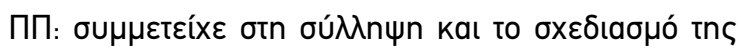

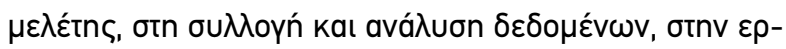

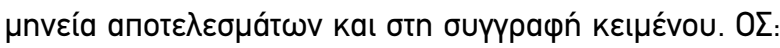

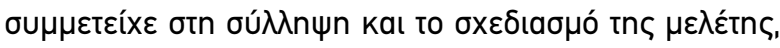

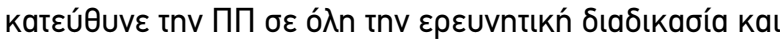

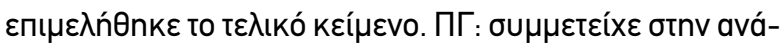
птU६n

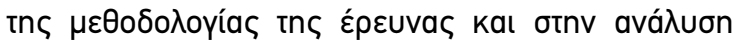

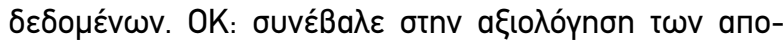

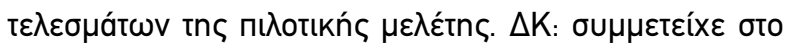

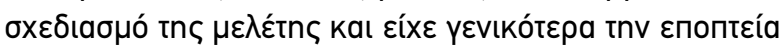

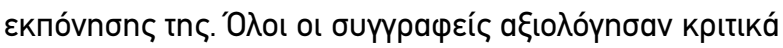

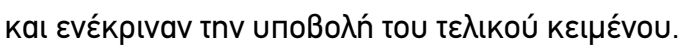

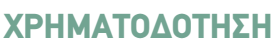

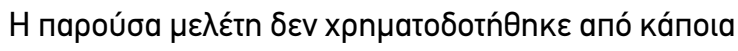
пnүń.

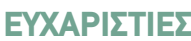

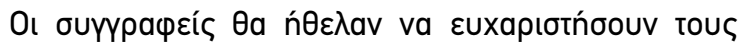

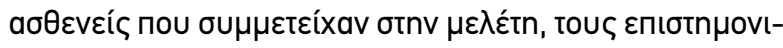

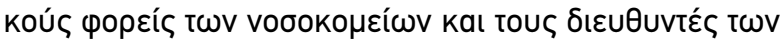

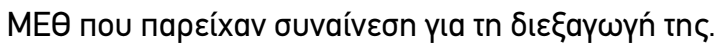




\section{EPEYNHTIKA APOPA}

\section{ВІВАІОГРАФІА}

Anhang Price R., Elliott M. N., Zaslavsky A. M., Hays R. D., Lehrman W. G., Rybowski L. \& Cleary P. D. (2014). Examining the role of patient experience surveys in measuring health care quality. Medical Care Research and Review 71(5):522-554.

Busico M., Intile D., Sívori M., Irastorza N., Alvarez A. L., Quintana J. \& Desmery P. (2016). Risk factors for worsened quality of life in patients on mechanical ventilation. A prospective multicenter study. Medicina intensiva 40(7):422-430.

Callahan L. A. \& Supinski G. S. (2016). Early Mobilization in the Intensive Care Unit: Help or Hype? Critical care medicine 44(6):1239-1240.

Chen, J. (2015). Integrated Care: Patient-Reported Outcome Measures and Patient-Reported Experience Measures: A Rapid Scoping Review. NSW Agency for Clinical Innovation, Sydney.

Engwall M., Fridh I., Johansson L., Bergbom I. \& Lindahl B. (2015). Lighting, sleep and circadian rhythm: an intervention study in the intensive care unit. Intensive and Critical Care Nursing 31(6):325335.

Fernandez R. R., Cruz J. S. \& Mata G. V. (1996). Validation of a quality of life questionnaire for critically ill patients. Intensive Care Medicine 22(10): 1034-1042.

Ferrand N., Zaouter C., Chastel B., Faye K., Fleureau C., Roze H. \& Ouattara A. (2019). Health related quality of life and predictive factors six months after intensive care unit discharge. Anaesthesia Critical Care \& Pain Medicine 38(2): 137-141.

Fildissis G., Zidianakis V., Tsigou E., Koulenti D., Katostaras T., Economou A. \& Baltopoulos G. (2007). Quality of life outcome of critical care survivors eighteen months after discharge from intensive care. Croatian medical journal 48(6):814-821.

Granja C., Lopes A., Moreira S., Dias C., Costa-Pereira A. \& Carneiro A. (2005). Patients' recollections of experiences in the intensive care unit may affect their quality of life. Critical Care 9(2):96-109.

Griffiths J., Hatch R. A., Bishop J., Morgan K., Jenkinson C. Cuthbertson B. H. \& Brett S. J. (2013). An exploration of social and economic outcome and associated health-related quality of life after critical illness in general intensive care unit survivors: a 12-month follow-up study. Critical care 17(3):R100.

Hofhuis J. G., Spronk P. E., van Stel H. F., Schrijvers A. J., Rommes J. H. \& Bakker J. (2008a). Experiences of critically ill patients in the ICU. Intensive and Critical Care Nursing 24(5):300-313.

Hofhuis J. G., Spronk P. E., van Stel H. F., Schrijvers G. J., Rommes J. H. \& Bakker J. (2008b). The impact of critical illness on perceived health-related quality of life during ICU treatment, hospital stay, and after hospital discharge: a long-term follow-up study. Chest 133(2):377-385.

Karabatsou D., Tsironi M., Tsigou E., Boutzouka E., Katsoulas T. \& Baltsopoulos G. (2016). Variable cost of ICU care, a micro-costing analysis. Intensive and Critical Care Nursing 35:66-73.

Kingsley C. \& Patel S. (2017). Patient-reported outcome measures and patient-reported experience measures. Bja Education 17(4):137144.

Lizana F. G., Bota D. P., De Cubber M. \& Vincent J. L. (2003). Long-term outcome in ICU patients. Intensive care medicine 29(8):1286-1293.

McKinley S., Fien M., Elliott R. \& Elliott D. (2016). Health-related quality of life and associated factors in intensive care unit survivors 6 months after discharge. American Journal of Critical Care 25(1):52-58
OECD (2017a). Health at a glance 2017. OECD INDICATORS. [Online]. Available at: http://www.oecd.org/health/health-systems/healthat-a-glance-19991312.htm [Accessed 10 March 2018]

OECD (2017b). Recommendations to OECD Ministers of health from the high level reflection group on the future of health statistics. Strengthening the international comparison of health system performance through patient-reported indicators. [Online]. Available at: https://www.oecd.org/els/health-systems/ Recommendations-from-high-level-reflection-group-on-thefuture-of-health-statistics.pdf [Accessed 03 March 2018]

Pandian V., Feller-Kopman D. \& Mirski M. (2013). 350: QUALITY OF LIFE IN MECHANICALLY VENTILATED INTENSIVE CARE UNIT PATIENTS. Critical Care Medicine 41(12):A82-A83.

Samuelson K.A. (2011). Unpleasant and pleasant memories of intensive care in adult mechanically ventilated patientsFindings from 250 interviews. Intensive and Critical Care Nursing 27(2):76-84.

Schenk P., Warszawska J., Fuhrmann V., König F., Madl C. \& Ratheiser K. (2012). Health-related quality of life of long-term survivors of intensive care: changes after intensive care treatment. Wiener klinische Wochenschrift 124(17-18):624-632.

Soliman I. W., de Lange D. W., Peelen L. M., Cremer O. L., Slooter A. J., Pasma W. \& van Dijk D. (2015). Single-center large-cohort study into quality of life in Dutch intensive care unit subgroups, 1 year after admission, using EuroQoL EQ-6D-3L. Journal of critical care 30(1):181-186

Stanchina M. L., Abu-Hijleh M., Chaudhry B. K., Carlisle C. C. \& Millman R. P. (2005). The influence of white noise on sleep in subjects exposed to ICU noise. Sleep medicine 6(5):423-428.

Stewart J. A., Green C., Stewart J. \& Tiruvoipati R. (2017). Factors influencing quality of sleep among non-mechanically ventilated patients in the intensive care unit. Australian Critical Care 30(2):85-90

Usta Y. Y., Dikmen Y. \& Başaran H. (2016). Factors Which Affect Patients' Experience in Intensive Care Units. International Journal of Health Sciences and Research 6(12): 143-149.

van der Schaaf M., Beelen A., Dongelmans D. A., Vroom M. B. \& Nollet F. (2009). Functional status after intensive care: a challenge for rehabilitation professionals to improve outcome. Journal of rehabilitation medicine 41(5):360-366.

Van Oostrom S. H., Gijsen R., Stirbu I., Korevaar J. C., Schellevis F. G., Picavet H. S. J. \& Hoeymans, N. (2016). Time trends in prevalence of chronic diseases and multimorbidity not only due to aging: data from general practices and health surveys. PloS one 11(8): $\mathrm{e} 0160264$.

Wåhlin I., Samuelsson P. \& Ågren S. (2017). What do patients rate as most important when cared for in the ICU and how often is this met?: An empowerment questionnaire survey. Journal of critical care 40:83-90.

Wehler M., Martus P., Geise A., Bost A., Mueller A., Hahn E. G. \& Strauss, R. (2001). Changes in quality of life after medical intensive care. Intensive care medicine 27(1):154-159.

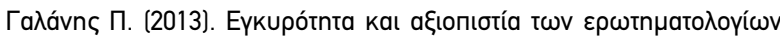

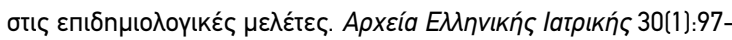
110 


\title{
Patients' reported experiences and Health Related Quality of Life after discharge from Intensive Care Units
}

Panagiota Peleka', Olga Siskou², Petros Galanis 3 , Olympia Konstantakopoulou'4, Daphne Kaitelidou ${ }^{5}$

1. CCRN, MSc, Intensive Care Department, 401 Military Hospital of Athens

2. RN, MSc, PhD, Senior Researcher, Center for Health Services Management and Evaluation, Nursing Department, National and Kapodistrian University of Athens

3. RN, MSc, PhD, Laboratory and Teaching Staff, Center for Health Services Management and Evaluation, Nursing Department, National and Kapodistrian University of Athens

4. Economist, MSc, PhD (c), Center for Health Services Management and Evaluation, Nursing Department, National and Kapodistrian University of Athens

5.Associate Professor, Center for Health Services Management and Evaluation, Nursing Department, National and Kapodistrian University of Athens

\begin{abstract}
Introduction: Providing high quality care in todays' Intensive Care Units is a challenge because of the constant increase in demand, the high functional cost and the restricted availability of resources. Evaluating both patients' experiences and outcomes from the care provided is necessary for ensuring high quality in health care.

Aim: The investigation of patient reported experiences in Intensive Care Units and the evaluation of their health related quality of life six to twelve months after discharge.

Methods: It is a non-interventional cross-sectional study with retrospective data collection. The participants were 108 patients, discharged from four Intensive Care Units of three military hospitals in Athens. Telephone interviews were conducted using "Patient Empowerment Questionnaire" and "Quality of life Questionnaire" to evaluate patients' experiences and health related quality of life respectively. Statistical analysis was conducted using the statistical package SPSS v. 25.0 .

Results: The majority of the study population reported positive experiences with the critical care staff and normal quality of life after discharge. However, $47 \%$ reported that they could not always sleep at night and $32 \%$ did not always receive adequate pain relief. In regards to patients' quality of life, "normal daily activities" were affected the most. Specifically, half of the patients reported inability to carry out activities requiring high level of physical effort and 1/5 expressed difficulties in walking and in mobility. Additionally, $43 \%$ reported difficulties in the job or their daily activities, have changed or completely abandoned them.

Conclusions: The patients' positive experiences and good health related quality of life after discharge gave the impression that quality of intensive care in Greek military hospitals is high. Interventions intending to achieve more adequate pain relief, better sleep at night, and prevention of critical illness' negative impact on day-to-day activities would ensure more positive results.
\end{abstract}

Key words: Critically ill patients, HRQOL, ICU, patient reported experiences and outcomes, quality of healthcare.

Correspondence: Panagiota Peleka

E-mail: panagiotapeleka@gmail.com

Submission date: 24/05/2019

Publication date: October 2020

Citation: Peleka P., Siskou O., Galanis P., Konstantakopoulou O. \& Kaitelidou D. (2020). Patients' reported experiences and Health Related Quality of Life after discharge from Intensive Care Units. Hellenic Journal of Nursing Science 13(4): 51-59, https://doi.org/10.24283/hjns.202045 